\title{
OPTIMIZATION OF PULSED LASER BASED METAL CLEANING PROCESS
}

\author{
Daniela Doina Cioboata ${ }^{1}$, Mihai Selagea ${ }^{2}$, Silvia Savencu², Radu Mihail Udrea², Mircea Virgil Udrea², \\ Dănuț Iulian Stanciu ${ }^{1}$, Valentin Șerban Popescu ${ }^{1}$ \\ ${ }^{1}$ National Institute of Research and Development for Mechatronics and Measurement \\ Technique - INCDMTM, Bucharest, Romania \\ Email:cioboatadoina@yahoo.com,danutstanciu@yahoo.com,valentinpopescu1995@gmail.com \\ ${ }^{2}$ Apel Laser srl, Str. Vanatorilor nr.25, 077135 Mogosoaia, Romania \\ Emails: mihai.selagea@apellaser.ro, silvia.savencu@apellaser.ro, radu.udrea@apellaser.ro, \\ mircea.udrea@apellaser.ro
}

\begin{abstract}
The cleaning of metallic surfaces offers the possibility to reuse old components or to prepare components for processes such as painting, thermal treatment, etc. There are three main methods to clean metallic surfaces: chemical, mechanical and by using laser beam. The chemical method is expensive, time consuming and polluting, the mechanical one has similar disadvantages, hence laser cleaning is the most efficient of them and it is increasingly more used in industrial applications. In our work we use a $100 \mathrm{~W}$ fiber laser based device that includes the laser itself, an expander, a scanner and a variable focus device. The influence of the laser parameters (power, pulse repetition rate, fluence) on the quality of the surface (conductivity and adherence) has been studied and optimized. A possibility to decrease the required laser power in specific cases is envisaged. Cleaning of 3D surfaces is considered.
\end{abstract}

Keywords: Metal Cleaning, Laser Cleaning, Laser Material Processing, Pulsed Laser, Laser Parameters.

\section{Introduction}

Different methods for cleaning of metallic surfaces (chemical, mechanical, or laser based) are used [1][8]. Chemical cleaning uses solvents to eliminate the organic or inorganic debris from the metallic surfaces. This method is polluting, the process duration is long and the cost is high. The environment is deeply affected. Mechanical cleaning is done by using abrasive materials such as sand that could deform the surface and it is very loud. The environment is polluted both physically and audibly. A better mechanical method is by using dry ice pellets. The production of pellets from liquid carbon dioxide is well known, especially in the food industry. A great advantage of using ice pellets is that it is a non poluting procedure. The pellets melt spontaneously and it has no impact on the environment. A pelletizer equipment is need in order to have a continuous source of pellets. Nevertheless, overall, the price is considerable.

Laser cleaning is the most convenient option in many cases $[2,3,5,9]$. A pulsed laser beam is focused on the metallic surface and a scan of the surface is performed. The evaporated material is removed immediately. This method is non-polluting, silent and fast. The price of the equipment and maintenance is recovered in a few years.

After laser processing, the surface is well prepared to be painted. So, large areas of stained metals could extend their lifetime. A good example is the laser cleaning of ships. The floating part of the ships is in permanent contact with sea water. Preventing corrosion by painting is very important.

Another example is cleaning surfaces to get the best possible electrical contact. This is the case in aerospace industry, where the electrical contact must be very good and the metallic surface (aluminium in this case) must be highly conductive where electrical pathways are connected.

In the last decades several studies have been done to identify the optimum working conditions for laser cleaning of different materials. Most of them were focused on cleaning and conservation of archeological artefacts [4], interaction between laser energy and surface layers [6], different types of laser used for surfaces cleaning. Other works focused on finding the effect of wavelength, fluence, pulse repetition rate and environment of laser cleaning for different applications [3][4], the mechanism of laser removal technology [7].

However, the analyses presented in the literature do not provide enough information on 
morphological changes that occur during the cleaning of various metal surfaces with laser, nor the effects of each process parameter on the surface characteristics after laser processing. In order to obtain the desired surface properties and avoid damage, a knowledge on the influence of each parameter on surface characteristics after laser processing is very important.

In this paper we perfom some studies in order to optimize the cleaning process by varying the laser parameters of a $100 \mathrm{~W}$ average power fiber laser.

\section{Experimental Set-Up}

Our prototype CLEAN100 ME is based on a $100 \mathrm{~W}$ average power fiber laser. The experimental device is shown in Figure 1 . The rate of repetition is adjustable between $30 \mathrm{kHz}$ and $200 \mathrm{kHz}$. By using the varioscan we are able to vary the position of the focus which could be useful for 3D cleaning.

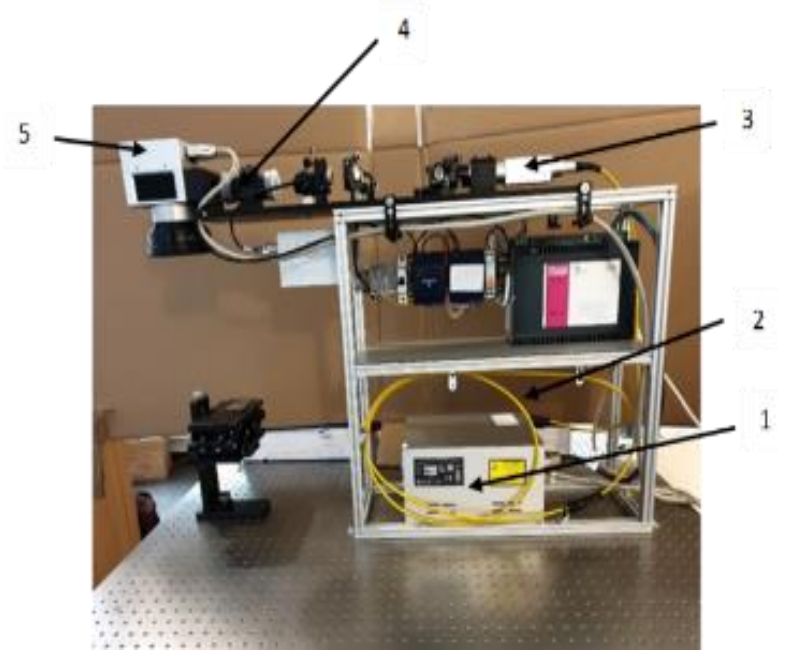

Figure 1: Experimental set up (1-fiber laser and laser power supply; 2 - transmission optical fiber;

3 - telescope; 4 -varioscan; 5 - optical scanner)

In Figure 2. the relationship between fiber laser average power and the repetition rate is given.

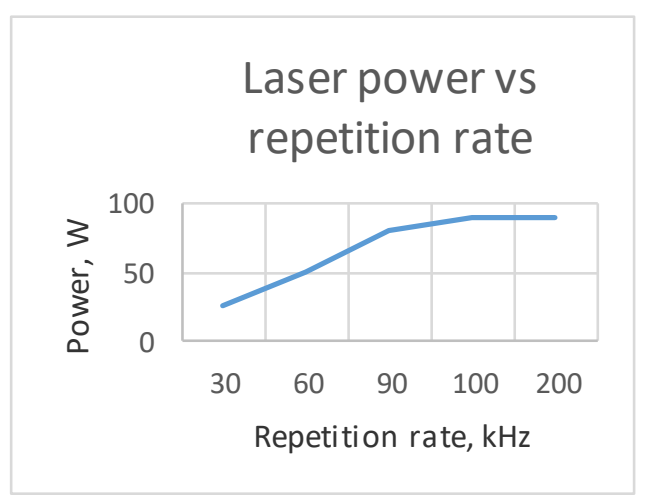

Figure 2: Laser average power versus repetition rate
The output average power is almost linearly depending on the repetition rate. By increasing the repetition rate from $30 \mathrm{kHz}$ to $200 \mathrm{kHz}$ the power is increasing up to a maximum value of about $95 \mathrm{~W}$ at $99 \mathrm{kHz}$ and afterwards it shows plateaus.

The laser power has been measured using a LabMax instrument and a PM-150 detector manufactured by Coherent Inc.

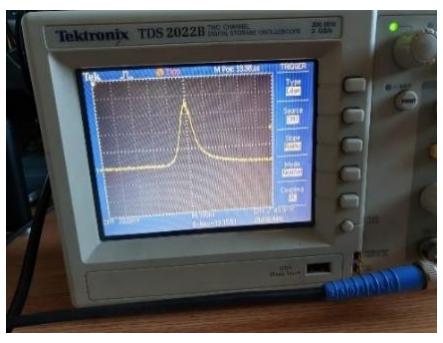

$a$.

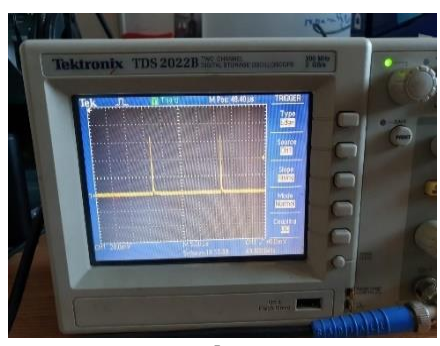

$b$.

Figure 3: Time behaviour of the laser pulses: single pulse (a), repetitive pulses (b).

The laser pulse is given in Figure 3. A fast detector (DET025A/M, Thorlabs) and a Tektronix TBS1042 oscilloscope were used. As shown in Figure 3, it results that the energy per pulse is aproximately $4 \mathrm{~mJ}$ and the peak pulse power is 10 MW, considering a duration of the laser pulse of 40 ns. The coresponding peak power density is aproximately $1.3 \cdot 10^{9} \mathrm{~W} / \mathrm{cm}^{2}$.

Focusing such an intense laser beam onto the surface to be cleaned leads to a breakdown on the surface. This consists of an evaporation of the unwanted material and a rejection of this material towards the laser beam. An exhauster is used to take off the debris. Since the base material (steel, aluminium) is reflecting back the laser beam (as it is below its plasma frequency) and a very small fraction is absorbed, a clean surface of the base material is obtained.

\section{Results}

Different materials were tested (EN S235JR steel, painted stainless steel, aluminium and dibond). The goal is to get a clean uniform surface and, in case of aluminium and dibond, a higher electrical conductivity path on the surface.

\section{$>$ Steel EN S235JR strongly oxidized}

We performed several tests on EN S235JR steel that is strongly oxidised. The microscopic aspect of 
the surface prior the cleaning process is given in Figure 4.

The chemical composition of the steel used is 0.2 C, $1.4 \mathrm{Mn}, 0.045 \mathrm{P}, 0.045 \mathrm{~S}$, balance $\mathrm{Fe}$, as maximum percentages in mass unit. The dimensions in figure 4 are $9 \times 9 \mathrm{~mm}^{2}$.

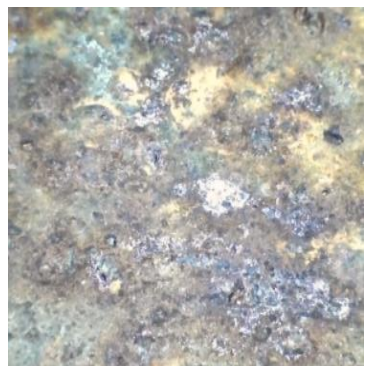

Figure 4: Microscopic structure of the steel piece, prior cleaning

In Figure 5 there are different rectangular regions which were exposed to the laser radiation at different powers $30 \mathrm{~W}, 60 \mathrm{~W}$ and $90 \mathrm{~W}$, from left to right, respectively.

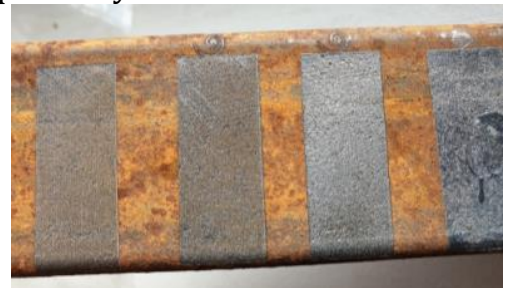

Figure 5: Removal of surface oxide on steel (width $40 \mathrm{~mm}$ )

Table 1. Results of varying power and repetition rate on surface oxide removal

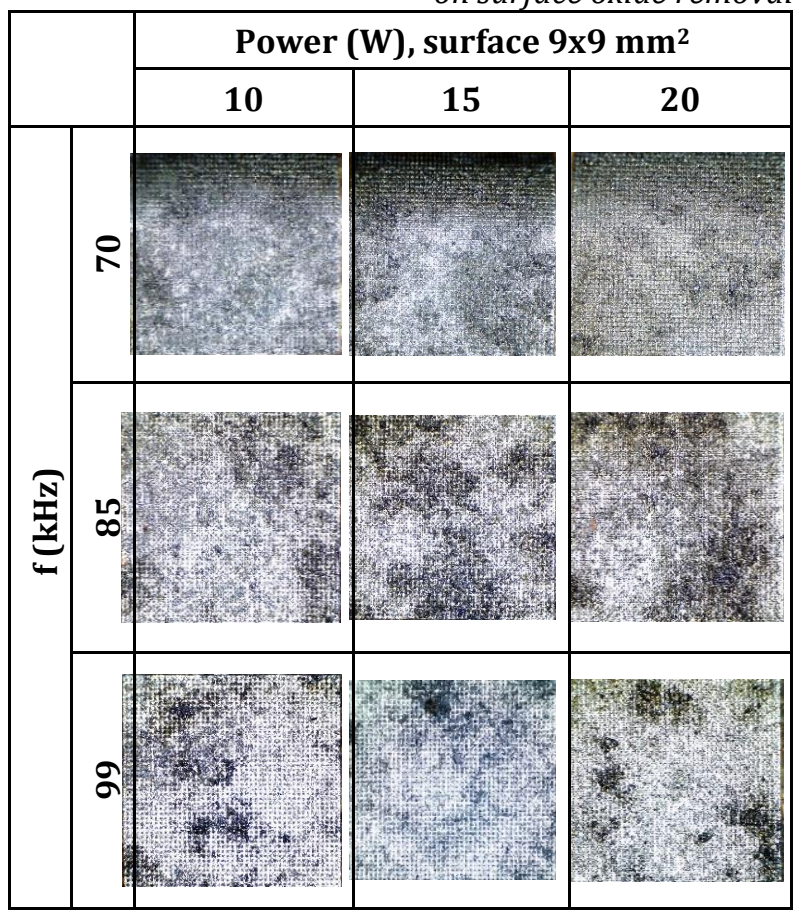

Table 1. shows the microscopic aspects of the surface for different parameters. The parameters considered were: laser average power, pulse repetition rate and scanning speed. The scanning speed has been kept constant $(0.1 \mathrm{~m} / \mathrm{s})$ as well as the line spacing $(0.2 \mathrm{~mm})$. In this case a satisfactory result was obtained at $15 \mathrm{~W}$ average power and 99 $\mathrm{kHz}$ repetition rate (see table 1 ). The irradiated surface is $9 \times 9 \mathrm{~mm}^{2}$.

The evolution of the adherence of the surface prior and after processing is evaluated. As predicted, the stained surface has a roughness that offers a better adherence than the cleaned surface, possibly linked to the increased effective surface area of contact. The simple experiment performed is shown in Figure 6.

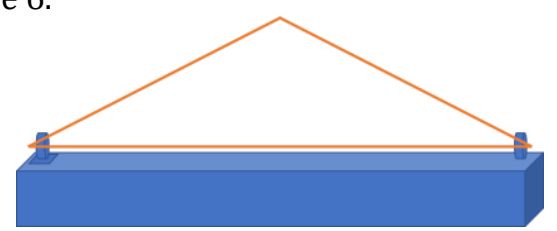

Figure 6: Experiment to determine the adherence.

Two small pieces were glued on the surface and a string was used to connect them. The time that is necessary for the steel piece to drop was measured. When the glue was applied on a stained surface the time was $1.1 \mathrm{~s}$. When the glue was applied on a cleaned surface the piece dropped instantly suggesting that the rough surface offers a bigger adherence.

\section{$>$ Painted stainless steel sheet}

Paint from a stainless steel 316L surface has been also removed using our set-up. In Figure 7 the surface structure prior to cleaning is shown.

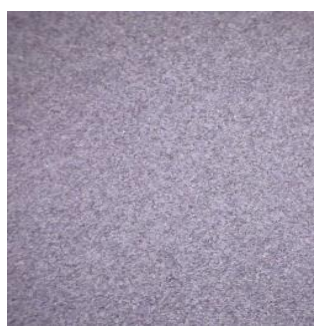

Figure 7: Stainless sheet prior to cleaning $\left(9 \times 9 \mathrm{~mm}^{2}\right)$

The scanning speed of $1.4 \mathrm{~m} / \mathrm{s}$ has been kept constant. Table 2 . shows the dependence of the cleaned surface aspect on laser power and repetition rate of the laser pulses. The irradiated surface is $9 \times 9$ $\mathrm{mm}^{2}$.

An experiment has been performed in order to use a laser with lower nominal power (equivalent of a cheaper laser). By individual spot irradiation in a matrix structure, the focused high intensity laser evaporates the paint and the clean reflective metallic surface is revealed. Between the irradiated surfaces, non-irradiated material was warmed by thermal conduction, so an easy manual peel of the non irradiated parts lead to a perfect surface. In this manner a good quality final surface is obtained by using a much cheaper laser, for example a $20 \mathrm{~W}$ laser instead of a $100 \mathrm{~W}$ laser. 
Table 2. Removal of paint from stainless steel sheet
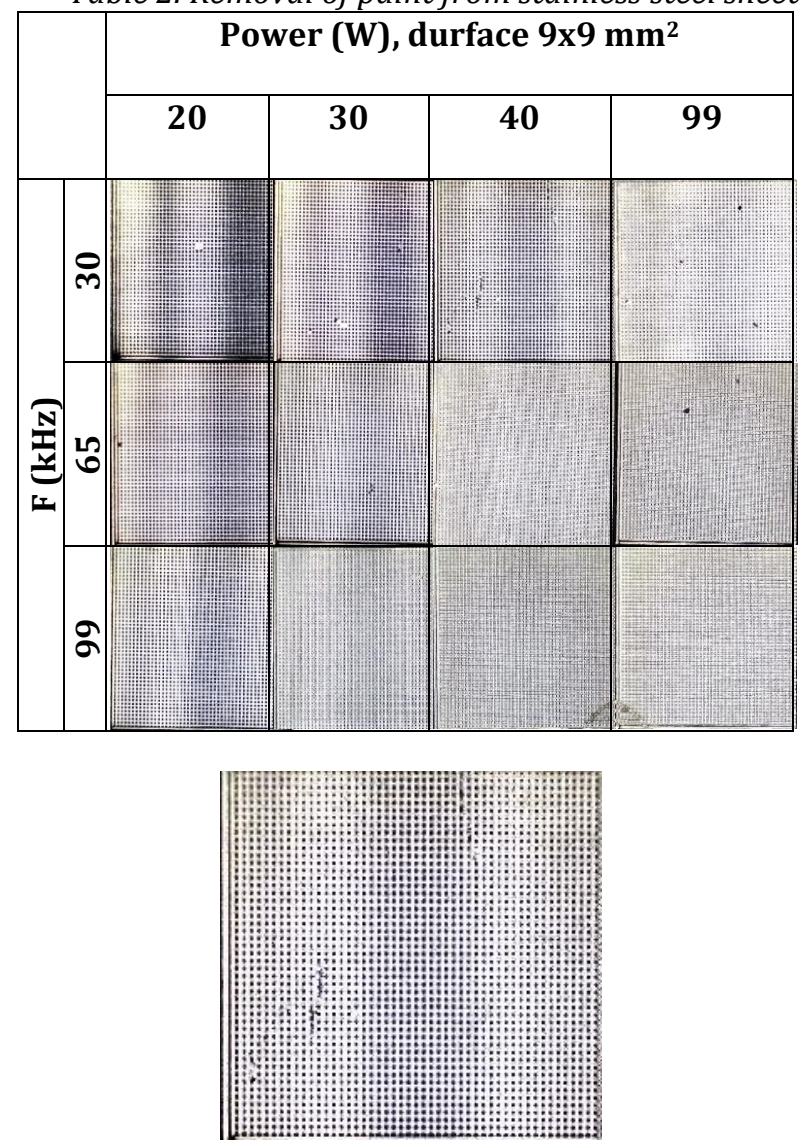

Figure 8: Matrix punctual aspect of the laser irradiation $\left(9 \times 9 \mathrm{~mm}^{2}\right)$

\section{Painted steel sheet}

A black painted steel sheet was exposed to laser cleaning process.

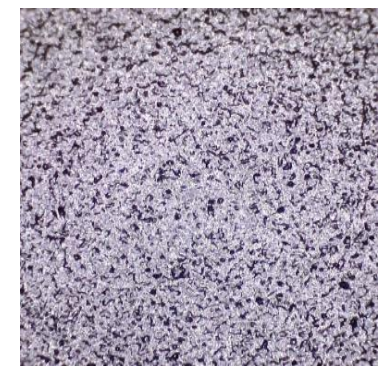

Figure 9: Initial microscopic image of the painted steel sheet $\left(9 \times 9 \mathrm{~mm}^{2}\right)$

The tests performed show that the best results were obtained with the following parameters: Power $=65 \mathrm{~W}$, Frequency $=65 \mathrm{kHz}$ (see table 3 ).

At higher powers and frequencies, the steel under the paint layer is burned. For a good cleaning of the paint layer avoiding thermal degradation of the material, two perpendicular passes were made, with the same parameters (Power $=65 \mathrm{~W}$, Frequency $=65$ $\mathrm{kHz}$ ), but with different speeds, the second pass being performed with higher speed.
Table 3. Painted steel sheets after irradiation for different parameters (speed $0.5 \mathrm{~m} / \mathrm{s}$ )

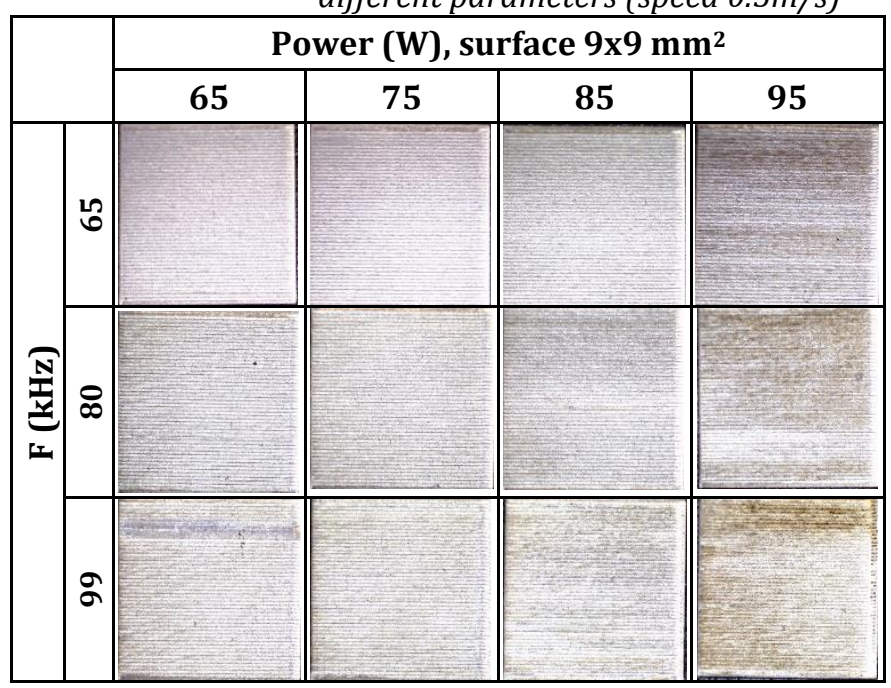

The microscopic aspect of the surface after cleaning is shown in figure 10 .

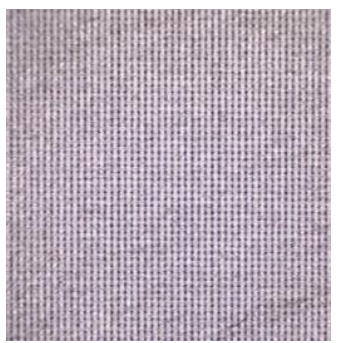

Figure 10: Microscopic aspect of the steel surface after cleaning the paint layer by two passes in ortogonal directions with the following parameters:

Pass $I-$ Power $=65 \mathrm{~W}$, Frequency $=65 \mathrm{kHz}$, Distance between lines $=0,18 \mathrm{~mm}$, Speed $=0,5 \mathrm{~m} / \mathrm{s}$;

Pass II - Power $=65 \mathrm{~W}$, Frequency $=65 \mathrm{kHz}$, Distance between lines $=0,18 \mathrm{~mm}$, Speed $=0,9 \mathrm{~m} / \mathrm{s}$

\section{$>$ Dibond}

Dibond is a composite material that consists of two aluminium plates and a polyethylene layer between them. It is largely used in the advertising industry. The aluminium is $0.3 \mathrm{~mm}$ wide and a $20 \mu \mathrm{m}$ strate of paint is covering it. In this case we intend to remove the painting and to obtain a high electrically conductive surface.

The dibond surface prior to cleaning is shown in figure 11 .

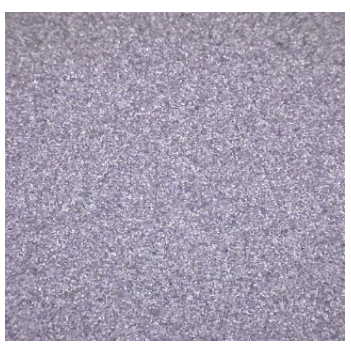

Figure 1: Dibond surface prior to cleaning $\left(9 \times 9 \mathrm{~mm}^{2}\right)$ 
Table 4. shows the microscopic aspects of the surfaces cleaned with different parameters.

Table 4. Removal of paint from the dibond surface, $9 \times 9$

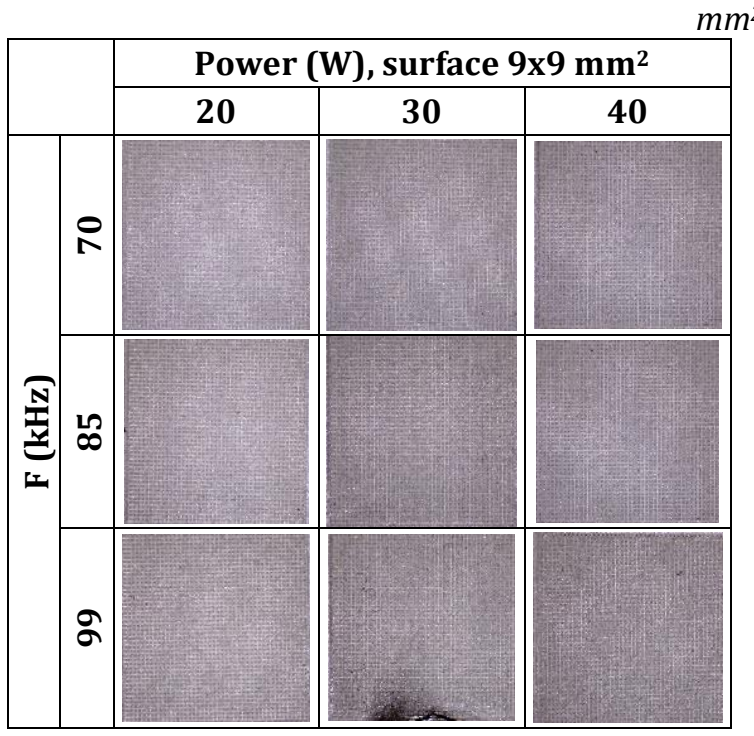

Initially, the dibond surface is not conductive because the protective layer of paint. After laser cleaning, the surface is highly conductive, a LED is emitting as shown in Figure 12. This application might be very useful when dibond plates are to be used in illumination panels and some conductive channels are necessary.

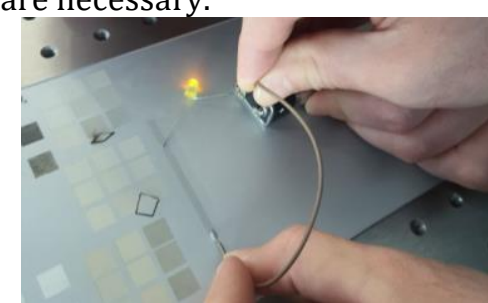

Figure 12: LED emission after removal of paint layer.

\section{Conclusions}

By using our prototype CLEAN100 ME we succesfully removed stains from stained steel sheet, aluminium oxide from alumnium plates, greases from stainless steel surfaces.

A set of measurements were performed in order to determine the optimal parameters.

Using an smaller average power laser to create a matrix of spots and a final manual cleaning step is a more economically efficient method in some cases.

Acknowledgements: This work was performed in the frame of the project ID P_40_390, SMIS 105552, subsidiary contract $2790 / 2018$ - Research on the realization of a fiber laser installation for microprocessing of metallic, ceramic and plastic materials, integrated within emerging processing technologies in the automotive industry - IMPLA, funded from the European Regional Development Fund, under the Competitiveness Operational Program 2014-2020, implemented by the Managing Authority within the Romanian Ministry of Investments and European Projects.

\section{References}

[1] Chandrasekar M, A Short Review on Alternative Cleaning Methods to Remove Scale and Oxide from the Jet Engine Alloys, International Journal of Engineering Research \& Technology (IJERT) ISSN: 2278-0181, Vol. 4 Issue 12, December-2015

[2] Mohammad Khairul Azhar Abdul Razab, An'amt Mohamed Noor, Mohamad Suhaimi Jaafar, Nor Hakimin Abdullah, Fatanah Mohamad Suhaimi, Mazlan Mohamed, Noraina Adam, Nik Alnur Auli Nik Yusu, A review of incorporating Nd:YAG laser cleaning principal in automotive industry, Journal of Radiation Research and Applied Sciences 11 (2018), pp 393-402

[3] Xiaoguang Li1, Tingting Huang, Ang Wei Chong, Rui Zhou, Yoo Sang Choo, Minghui Hong, Laser cleaning of steel structure surface for paint removal and repaint adhesion, Opto-Electronic Engineering, 2017, Vol 44, Issue 3, pp 340-344

[4] Vishnu Narayanan, Ramesh Kumar Singh, Deepak Marla, Laser cleaning for rust removal on mild steel: An experimental study on surface characteristics, MATEC Web of Conferences 221, ICDME

2018, DOI: $10.1051 /$ matecconf/201822101007

[5] V. P. Veiko, T. Ju. Mutin, V. N. Smirnov, E. A. Shakhno, S. A. Batishche, Laser cleaning of metal surfaces: physical processes and applications, Proceedings Volume 6985, Fundamentals of Laser Assisted Micro- and Nanotechnologies; 69850D (2008) https://doi.org/10.1117/12.786975

[6] Mottner P., Wiedemann G., Haber G., Conrad W., Gervais A. (2005) Laser Cleaning of Metal Surface - Laboratory Investigations. In: Dickmann K., Fotakis C., Asmus J.F. (eds) Lasers in the Conservation of Artworks. Springer Proceedings in Physics, vol 100. Springer, Berlin, Heidelberg. https://doi.org/10.1007/3540-27176-7_10

[7] Yanqun Tong, Furong Li, Jianyu Huang, Xiaoyi Wu, Xudong Ren, Simulation and Research on the Laser Removal Mechanism, International Journal of Nonlinear Science Vol.23(2017) No.3, pp.137141, ISSN 1749-3889 (print), 1749-3897 (online)

[8] Van-Mui Nguyen, Hai-Nam Duong, Minh- Duc Truong, VanHoa Dang, Viet-Cuong Chu, A Research on Optical Fiber Laser Interaction with Materials, Applied on Surface Cleaning of Mechanical Parts, International Journal of Scientific Engineering and Science, Volume3, Issue 10, pp.48-50,2019, ISSN (Online): 2456-7361

[9] Razab, M.K.A.A., Jaafar, M.S., Abdullah, N.H. et al., Influence of Elemental Compositions in Laser Cleaning for Automotive Coating Systems, Journal of Russian Laser Research, 37, 197-206 (2016). https://doi.org/10.1007/s10946-016-9561-0 